\title{
Magnetoexciton in vertically coupled InP/GaInP quantum disks: Effect of strain on the exciton ground state
}

\author{
K. L. Janssens, B. Partoens, * and F. M. Peeters ${ }^{\dagger}$ \\ Department Fysica, Universiteit Antwerpen (Campus Drie Eiken), Universiteitsplein 1, B-2610 Antwerpen, Belgium
}

(Received 22 May 2003; revised manuscript received 23 October 2003; published 25 June 2004)

\begin{abstract}
The influence of strain on the exciton properties in self-assembled two and three vertically coupled $\mathrm{InP} / \mathrm{GaInP}$ dots is investigated theoretically. For closely stacked dots, the strain fields arising from the different dots were found to interact with each other and modify the effective electron and hole potentials considerably. We found that the light hole state is the ground state for the systems under consideration and that we have a type-II configuration for the exciton. A comparison with experimental results for the exciton diamagnetic shift shows reasonable agreement (very good agreement for $B<20 \mathrm{~T}$ and for $B>20 \mathrm{~T}$, the quality of the agreement depends on the interdot distance) and confirms the earlier proposed experimental interpretation [M. Hayne et al., Phys. Rev. B 62, 10324 (2000)] that the light hole is located outside the dots. For closely spaced dots the light hole is above and below the stack of dots, while for a large vertical separation of the dots the light hole is located between the dots.
\end{abstract}

DOI: $10.1103 /$ PhysRevB.69.235320

PACS number(s): 73.21.La, 71.35.Ji, 85.35.Be

\section{INTRODUCTION}

Recently, there has been much interest in the study of quantum dots, which are structures that confine charge carriers in all three dimensions. Especially the selfassembled quantum dots ${ }^{1}$ are considered to be very promising for possible applications, such as quantum dot lasers, ${ }^{2}$ due to their large confinement energy and high optical quality.

The formation of such self-assembled dots requires two semiconductor materials with a substantially different lattice parameter, which are grown on top of each other. Such lattice-mismatched hetero-epitaxy gives rise to the formation of nanometer sized islands, governed by strainrelaxation effects, and is called "Stranski-Krastanow" growth.

The confinement of charge carriers in quantum dots occurs because of the difference in the bandstructure of the two semiconductor materials. One can define two types of quantum dots, namely type-I and type-II, depending on the band alignments. In the case of type-I dots, both the electron and the hole are confined inside the dot. For type-II dots, the confinement inside the dot occurs only for one of the charge carriers, i.e., electron or hole, whereas the dot forms an antidot for the other particle. Examples of type-II dots are the InP/GaInP ${ }^{3,4}$ and InP/GaAs dots with the electron confined in the dot and the hole in the barrier, and the $\mathrm{GaSb} / \mathrm{GaAs}^{5}$ and InAs $/ \mathrm{Si}^{6}$ dots where the hole is located inside the dot, but the electron remains in the barrier.

Up to now most research was devoted to type-I structures, ${ }^{7-12}$ while type-II dots have attracted less attention. Theoretical studies considering InP/GaInP dots were performed by Pryor et al. ${ }^{13}$ and Tadić et al. ${ }^{14}$ where a straindependent $\mathbf{k} \cdot \mathbf{p}$ Hamiltonian was used to calculate the electronic structure. The present authors studied the influence of external fields on single and vertically coupled InP/GaInP dots in the absence of strain. ${ }^{15,16} \mathrm{GaSb} / \mathrm{GaAs}$ quantum dots were investigated by Lelong et al. ${ }^{17}$ who studied excitons and charged excitons, and by Kalameitsev et al. ${ }^{18}$ who investigated the effect of a magnetic field.

The formation of self-assembled quantum dots is inextricably bound up with the occurrence of strain fields in and around the dots. The strain will have a large impact on the bandstructure, and hereby also on the optical properties of the dots. The hydrostatic component of the strain will shift the conduction and valence band edges, while the biaxial strain modifies the valence bands by splitting the degeneracy of the light- and heavy-hole bands.

Different theoretical models exist to obtain the strain distribution in and around self-assembled quantum dots. Wellknown and extensively used are the continuum mechanical (CM) model $^{9,14}$ and the valence force field (VFF) model. ${ }^{9,19,20}$ In the CM model the elastic energy is minimized to obtain the distribution of the displacement in the structure and the corresponding strain fields, whereas the VFF model is an atomistic approach, which uses phenomenological expressions for the elastic energy, depending on the atomic coordinates. Pryor et al. compared the two methods and found agreement for small strains, whereas for larger strains discrepancies were reported. ${ }^{21}$ This was corroborated very recently by Tadić et al., ${ }^{20}$ who also compared both methods but now for cylindrical InAs/GaAs and InP/InGaP dots, and found a better agreement for InP/GaInP than for InAs/GaAs dots, where the latter has the largest lattice mismatch.

A more simple method to calculate the strain fields near an isotropic quantum dot was presented by Downes et al., ${ }^{22}$ who used Eshelby's theory of inclusions ${ }^{23}$ to express the strain distribution in quantum dot structures. This approach was adapted by Davies, who showed that the elastic field can be derived from a scalar potential that obeys a Poisson equation with the lattice mismatch as charge density. ${ }^{24}$

In a previous study, ${ }^{25}$ we followed the work of Davies ${ }^{24}$ and used the so-called isotropic elasticity model to calculate the strain fields around a single cylindrical quantum disk. 


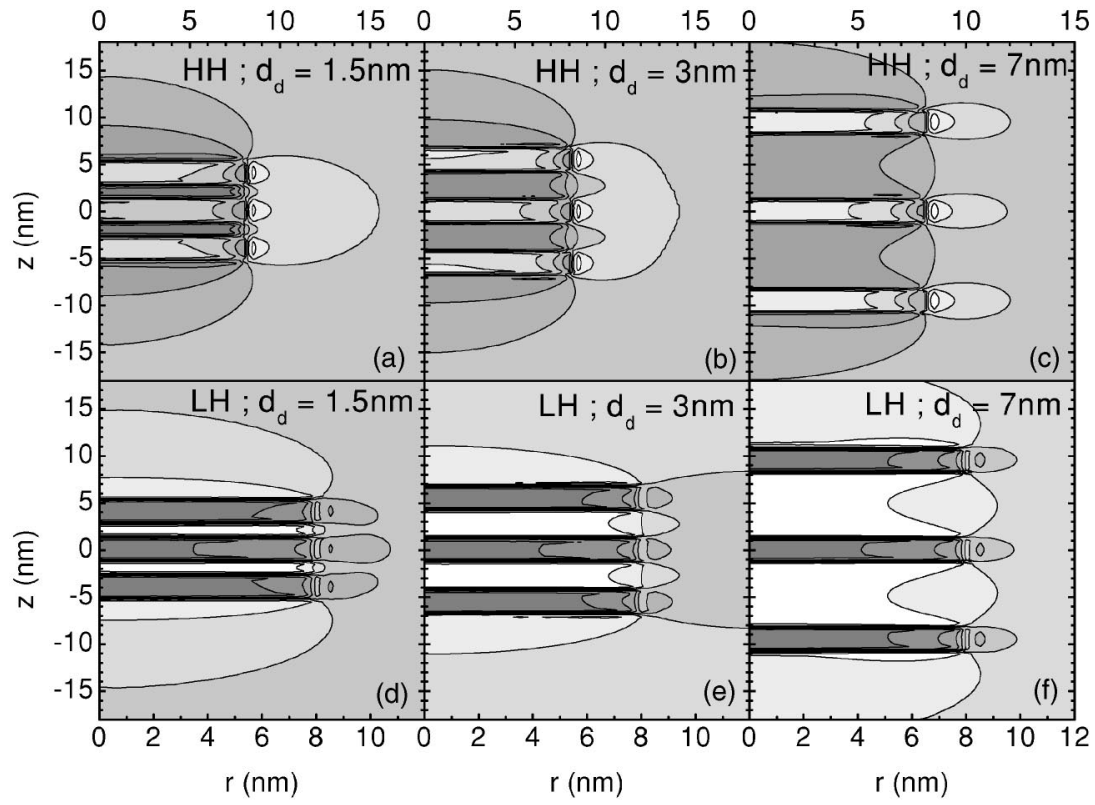

FIG. 1. Contour plots of the effective confinement potential for the heavy (a)-(c) and light (d)-(f) hole for, respectively, the interdot distances $d_{d}=1.5 \mathrm{~nm}, d_{d}=3.0 \mathrm{~nm}$, and $d_{d}=7.0 \mathrm{~nm}$, as indicated, for three vertically stacked identical dots with radius $R=8 \mathrm{~nm}$ and thickness $d=2.5 \mathrm{~nm}$. Dark colored regions denote lower potential values.
The strain distribution was used as an input to calculate the modification to the band structure and a single band effective mass approximation was applied to calculate the exciton properties in single InP/GaInP quantum disks in the presence of a perpendicular magnetic field, considering both heavyand light-hole bands. It was found that in flat InP/GaInP disks the heavy hole is the ground state while for more pillar like systems the light hole exciton has the lowest energy. The heavy hole is a type-I system while the light hole was found to be type-II. An "exciton" phase diagram for single InP/ GaInP disks was constructed.

In the present paper, we extend our previous approach to the study of two and three vertically coupled quantum disks. As previously, the isotropic elasticity model will be used to calculate the strain fields and the excitonic properties are calculated within the single band effective mass approximation. Special attention will be paid to the spatial location of the electron and hole in the system and the type of hole, i.e., heavy versus light, contributing to the ground state of the magneto-exciton. The magnetic field dependence of the exciton energy will be compared with the experimental magneto-luminescence experiments of Hayne et $a .^{3}$

The paper is organized as follows. In Sec. II, we explain our theoretical model. In Sec. III we discuss the influence of the strain on the confinement potential, while in Sec. IV we compare our numerical results with the experimental data of Ref. 3. Our results are summarized in Sec. V.

\section{THEORETICAL MODEL}

Our theoretical approach is similar to the one we used in Ref. 25. The strain is calculated by adapting the method used in Ref. 24, and which was described in Ref. 14. For the calculation of the exciton properties, a mean-field type of approach was used in the Hartree approximation. This approach was previously introduced by us for type-II quantum dots where strain ${ }^{16,26}$ was neglected. The coupled singleparticle equations are solved self-consistently. More information about the implementation and numerical procedure can be found in Refs. 25 and 26. Our numerical procedure is based on a two-dimensional finite difference method. Whereas in our previous studies we used a uniform space grid in two dimensions, for the present study we implemented a nonuniform mesh. This nonuniform mesh allows us to consider more gridpoints in regions where there is a strong variation of the wavefunction and at the same time consider a larger overall region in space. This is specifically important for the present study, as we expect the heavy hole to be strongly confined inside the disks, while the light hole will be more spread out above, below, and between the stack. $^{27}$

In our numerical calculation, we made use of the material parameters of InP/GaInP, as used in Refs. 13 and 14, which are also given in Table 1 of Ref. 25. From these data it follows that the valence band offset for this system is negative $\left(V_{h}=-45 \mathrm{meV}\right)$, i.e., we have a type-II system. In our previous study, we pointed out the possibility of dealing with a type-I system, ${ }^{28}$ but we showed that this has only a minor influence on the final results ${ }^{25}$ due to the small size of the band offset, i.e., corrections to $V_{h}$ due to strain effects are much more important.

\section{INFLUENCE OF STRAIN ON THE CONFINEMENT POTENTIAL FOR THREE VERTICALLY COUPLED DISKS}

In Ref. 25, we demonstrated that an increase of the quantum disk thickness can induce a type-I to type-II transition for the heavy hole. Such thick quantum dots are, however, difficult to realize experimentally. It is therefore interesting to investigate the properties of a vertical stack of thin quantum disks which may resemble such thick dots. Already in Ref. 16, we showed that the properties of a ver- 


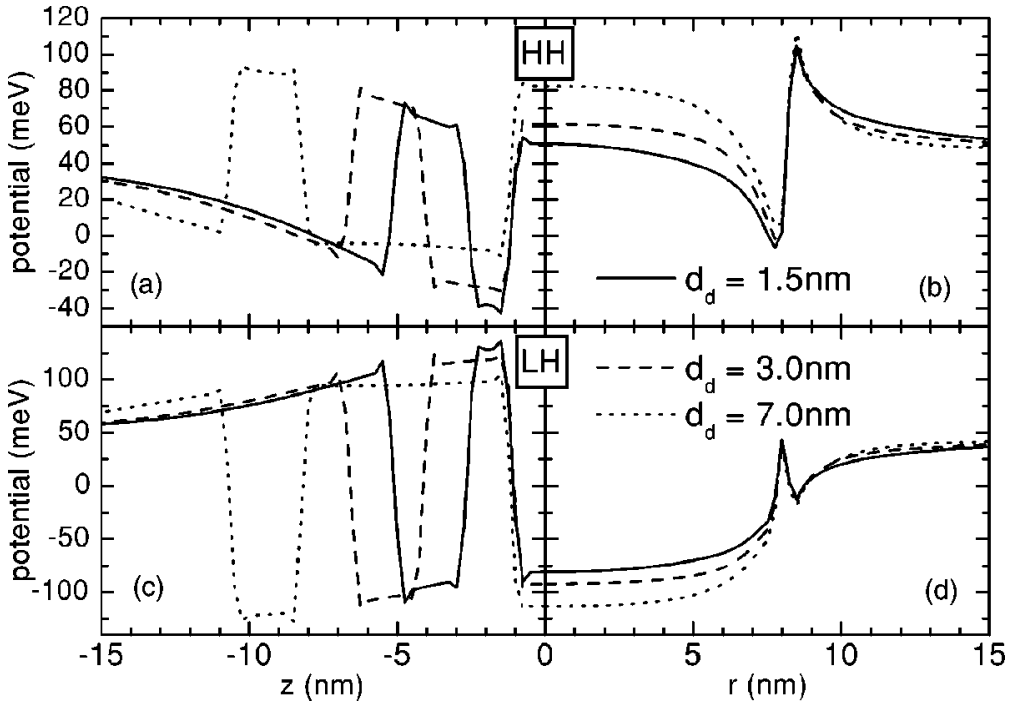

FIG. 2. The effective confinement potentials along the $z$-direction for $r=0$ [(a) and (c)] and along the radial direction for $z=0$ [(b) and (d)], both for the heavy (upper panels) and the light (lower panels) hole. The solid curve indicates the result for $d_{d}=1.5 \mathrm{~nm}$, whereas the dashed and dotted curves denote, respectively, the interdot distances $d_{d}=3 \mathrm{~nm}$ and $d_{d}=7 \mathrm{~nm}$ of the three vertically stacked dots with radius $R=8 \mathrm{~nm}$ and thickness $d=2.5 \mathrm{~nm}$. tical stack of unstrained dots are similar to those of a single dot with a thickness comparable to the stack height if the separation between the dots is sufficiently small. This study, however, did not include the strain fields in and around the dots.

Figure 1 shows the effective confinement potentials (i.e., geometric and strain confinement potentials) for both the heavy [upper row, (a)-(c)] and the light [lower row, (d)-(f)] hole, for respective interdot distances $d_{d}=1.5 \mathrm{~nm}, d_{d}$ $=3.0 \mathrm{~nm}$ and $d_{d}=7.0 \mathrm{~nm}$. The lighter regions denote higher potentials, i.e., regions where the hole prefers to sit. Here the effective potential denotes the total confinement due to strain, which is given by

$$
\begin{aligned}
& V_{h h}=V_{h}+a_{v} \varepsilon_{h y}+b\left(\left(\varepsilon_{x x}+\varepsilon_{y y}\right) / 2+\varepsilon_{z z}\right), \\
& V_{l h}=V_{h}+a_{v} \varepsilon_{h y}-b\left(\left(\varepsilon_{x x}+\varepsilon_{y y}\right) / 2+\varepsilon_{z z}\right),
\end{aligned}
$$

for, respectively, the heavy and the light hole. $V_{h}$ denotes the unstrained confinement potential, $a_{v}$ and $b$ are the valence band deformation potentials and $\varepsilon_{h y}$ is the hydrostatic strain. The radius and thickness of each of the disks were, respectively, taken as $R=8.0 \mathrm{~nm}$ and $d=2.5 \mathrm{~nm}$, corresponding to those studied in the experiment of Ref. 3 .

We find that a vertical stacking of dots has an appreciable influence on the strain distribution in and around the stack, and therefore also on the effective potential. In Ref. 25, we found that, starting from a negative unstrained valence band offset, the heavy-hole band offset becomes positive when strain is included while the light-hole band offset becomes more negative. Furthermore, for the heavy hole, we found a potential maximum at the radial boundary of the disk, which becomes relatively more important for increasing disk thickness.

Figures 1(a)-1(c) show the result for the heavy hole in the case of three vertically stacked quantum disks. For a closely stacked system, i.e., $d_{d}=1.5 \mathrm{~nm}$, we find that the potential maxima at the radial boundaries of the three dots merge almost into one big potential maximum and the potential approaches the one of a thick single disk. With increasing interdot distance, this merging decreases and for $d_{d}=7.0 \mathrm{~nm}$ we find only a small interaction between the dots, while the potential maxima at the radial boundaries remain distinguishable. The highest potential maximum is now found inside the disks.

Figure 2 shows cuts of the effective potentials of Fig. 1 along the $z$-direction ar $r=0$ (left panel) and along the $r$-direction for $z=0$ (right panel). For the heavy hole [Figs. 2(a) and 2(b)], we find that a closer stacking results in a decrease of the effective potential inside the dots, along both directions. Furthermore, we find analogous to the single disk-case that the potential maximum along the radial direction is not affected by a variation of the interdot distance, making it favorable for the heavy hole to move to this position for smaller interdot distances. For the light hole [Figs. 2(c) and 2(d)], we find that the highest potentials are found between the dots and above/below the stack. Along the radial direction there is also a potential maximum outside the disk boundary, but this is negligible compared to the potentials along the $z$-direction.

A consequence of the merging of the radial potential maxima for small interdot distances is that the heavy hole will prefer to move towards this maximum, which is higher than the potential maximum inside the disk. Moreover, the region of the radial maximum is broader and consequently the heavy hole will have a substantial larger space available then when sitting inside the disks. This is depicted in Fig. 3(a), where the heavy-hole probability density is depicted for $d_{d}=1.5 \mathrm{~nm}$ (the dark region denotes the highest probability density). When the heavy hole is sitting at the radial boundary in the barrier material, the system is type-II. This result is similar to what we found for single thick disks, i.e., for a pillar like system, in Ref. 25.

With increasing the interdot distance, the deep broad potential maximum at the radial boundary disappears and the potential maximum inside the dots becomes higher. This causes the heavy hole to move away from the radial boundary into the quantum disks, which is shown in Figs. 3(b) and 


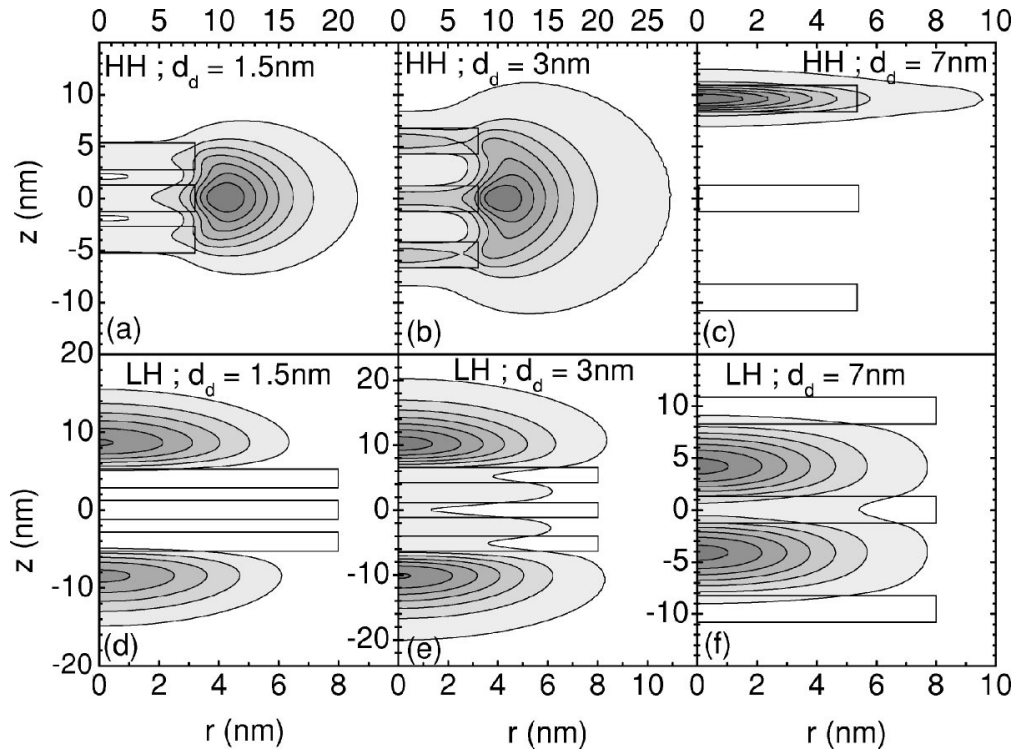

FIG. 3. Contour plots of the probability density for the heavy (a)-(c) and light (d)-(f) hole wave functions for, respectively, $d_{d}=1.5 \mathrm{~nm}, d_{d}$ $=3.0 \mathrm{~nm}$ and $d_{d}=7.0 \mathrm{~nm}$, as indicated. Dark colored regions denote a higher probability density. 3(c)). For $d_{d}=7 \mathrm{~nm}$ [Fig. 3(c)], the heavy hole ground state was found to be asymmetric: the heavy hole resides in the upper or lower disk. This asymmetry is induced by a higher potential for the heavy hole in the upper and lower disk (a consequence of strain), as found in Figs. 1, together with an increase of the Coulomb interaction energy for the asymmetric state. ${ }^{15,16}$ The actual heavy hole ground state is nevertheless a symmetric state along the $z$-direction which is a superposition of the one depicted in Fig. 3(c) and an equivalent one which resides in the bottom dot. Because of the large width of the tunnel barrier between the upper and lower disk, this gain in energy due to the symmetrization of the wavefunction is negligibly small. We can thus summarize that, in the case of vertically stacked quantum disks, a decrease of the interdot distance $d_{d}$ can cause a type-I to type-II transition for the heavy hole.

For the light hole, we find that the potential minimum is always inside the disk [Figs. 1(d)-1(f)]. The light hole (holes prefer to sit near the potential maxima) will thus prefer to remain in the barrier. The exact position, however, will also depend on the interdot distance. In the case of a small $d_{d}$, there is not enough space for the light hole to sit between the disks, and it will be located on top of and below the stack [Fig. 3(d)]. With increasing interdot distance, the probability to find the light hole between the disks also increases. Indeed, for $d_{d}=7.0 \mathrm{~nm}$, we find that the light hole sits completely between the disks [Fig. 3(f)].

\section{COMPARISON WITH EXPERIMENTS FOR THREE AND TWO VERTICALLY COUPLED DOTS}

In Ref. 3, results were reported of magnetophotoluminescence experiments on single and vertically stacked self-assembled InP/GaInP quantum dots. The measurements were done in magnetic fields up to $B=50 \mathrm{~T}$ using pulsed fields, and a model was proposed, suggesting that the holes are confined in the barrier material, whereas the electrons reside inside the disks.

Analogous to Ref. 25, we will disregard band mixing be- tween the heavy- and light-hole bands. To obtain the exciton energy, we thus solve two sets of coupled equations: one for the heavy-hole exciton and one for the light-hole exciton. In order to determine the ground state of the system, we compare the heavy- and the light-hole solution. The exciton energy is given by

$$
E_{\text {exciton }}=E_{e}+E_{h h(l h)}+\frac{e^{2}}{4 \pi \epsilon} \iint \frac{\rho_{e}(r, z) \rho_{h}\left(r^{\prime}, z^{\prime}\right)}{\left|\mathbf{r}-\mathbf{r}^{\prime}\right|} d \mathbf{r} d \mathbf{r}^{\prime},
$$

where $E_{e}$ is the single electron energy and $E_{h h(l h)}$ the single heavy- (light-) hole energy. The transition energy is defined as

$$
E_{\text {trans }}=E_{\text {exciton }}+E_{g}
$$

where $E_{g}$ is the band-gap energy of the disk material.

In a previous section, we showed that the strain, together with the interdot distance, has a large impact on the position of the wave functions in a system of three vertically coupled disks. We will now further investigate which is the ground state of the system, and how the exciton energy depends on the interdot distance and on the externally applied magnetic field $\mathbf{B}=B \mathbf{e}_{z}$. We study the systems with three coupled disks with respective interdot distances $d_{d}=1.5 \mathrm{~nm}, d_{d}=3.0 \mathrm{~nm}$, and $d_{d}=7.0 \mathrm{~nm}$, and also the system with two coupled disks, where $d_{d}=1.5 \mathrm{~nm}$. For all systems, the stacked disks are of equal dimension, namely disk radius $R=8 \mathrm{~nm}$ and thickness $d=2.5 \mathrm{~nm}$. Furthermore, we use the material parameters from Table I.

From the exciton transition energy, we find that in each system the light-hole exciton has a lower energy than the heavy-hole exciton. This is consistent with our previous study, ${ }^{25}$ where we showed that, for a single disk, the light hole becomes the ground state when the disks are sufficiently thick, i.e., for $R=8 \mathrm{~nm}$ when $d>4.5 \mathrm{~nm}$. 
TABLE I. Material parameters, taken from Ref. 14.

\begin{tabular}{lcc}
\hline \hline Parameter & $\mathrm{InP}$ & $\mathrm{Ga}_{0.51} \mathrm{In}_{0.49} \mathrm{P}$ \\
\hline$m_{e}^{*}\left(m_{0}\right)$ & 0.077 & 0.125 \\
$\gamma_{1}$ & 4.95 & 5.24 \\
$\gamma_{2}$ & 1.65 & 1.53 \\
$m_{h h, \|}$ & 0.1515 & 0.1477 \\
$m_{h h, z}$ & 0.606 & 0.4587 \\
$m_{l h, \|}$ & 0.303 & 0.269 \\
$m_{l h, z}$ & 0.121 & 0.1205 \\
$E_{g}(\mathrm{eV})$ & 1.424 & 1.97 \\
$V_{c}(\mathrm{eV})$ & 1.379 & 1.97 \\
$V_{v}(\mathrm{eV})$ & -0.045 & 0.0 \\
$a_{c}(\mathrm{eV})$ & -7.0 & \\
$a_{v}(\mathrm{eV})$ & 0.4 & \\
$b(\mathrm{eV})$ & -2.0 & 12.61 \\
$\epsilon$ & 12.61 & 0.56532 \\
$a(\mathrm{~nm})$ & 0.58687 & \\
\hline \hline
\end{tabular}

Because the thickness of the dots $d=2.5 \mathrm{~nm}$ is below the critical value $4.5 \mathrm{~nm}$, the heavy hole should become the ground state when the dots are far apart. We found that this occurs for $d_{d} \geqslant 15 \mathrm{~nm}$.

A comparison of the exciton energy with the experimental results of Ref. 3 shows that our theory tends to underestimate the experimental values. We attribute this to uncertainties regarding the disk and/or material parameters. In particular, a change of the disk thickness will lead to a uniform shift of the transition energy, i.e., it will not alter the magnetic field dependence. To investigate the magnetic field dependence of the exciton energy, we studied in more detail the diamagnetic shift. The diamagnetic shift gives the change of the exciton energy under the application of a magnetic field and is defined as

$$
\Delta E=E(B)-E(B=0) .
$$

In Fig. 3, the diamagnetic shift is plotted as a function of the magnetic field for, respectively, the systems with three vertically coupled disks with $d_{d}=1.5 \mathrm{~nm}$ (a), $d_{d}=3.0 \mathrm{~nm}(\mathrm{~b})$, and $d_{d}=7.0 \mathrm{~nm}$ (c), and the system with two vertically coupled disks with $d_{d}=1.5 \mathrm{~nm}(\mathrm{~d})$. Here the solid and dashed curves denote, respectively, our results for the heavy- and the light-hole exciton, while the squares indicate the experimental result by Hayne et al. $^{3}$

First, we consider the system with three coupled dots with interdot distance $d_{d}=1.5 \mathrm{~nm}$. The agreement between the light-hole exciton curve and the experimental result is very good, in particular in view of the fact that no fitting parameters are introduced in the theory. It is clear that experimentally the light-hole exciton is observed, which is located above and below the stack of dots [see Fig. 3(d)].

As we saw in Sec. III, for an interdot distance $d_{d}=1.5 \mathrm{~nm}$, the strain pushes the heavy hole towards the radial boundary of the stack. From our previous studies ${ }^{16,25,26}$ on a system where the hole is located at the radial boundary, we know that application of a magnetic field leads to angular momentum transitions of the hole angular momentum $l_{h}$. This is indeed also what we find in the present case for the heavy-hole exciton. The different solid curves in Fig. 4(a) show the result for, respectively, $l_{h}=0,1,2,3$ and 4 , as indicated. We find that the final heavy-hole ground state (consisting of different angular momentum states) approaches now more closely the light-hole result and the experimental result, although it is not the ground state of the system. No such angular momentum transitions were found experimentally, which is consistent with our previous conclusion that experimentally the light-hole exciton is observed.

The next system under investigation is the system where the interdot distance between the disks is $d_{d}=3 \mathrm{~nm}$. The result for the diamagnetic shift is shown in Fig. 4(b), where we find that the theoretical results for the light-hole exciton approximate closely the experimental result. Although the agreement is less than for $d_{d}=1.5 \mathrm{~nm}$, the discrepancy is at most only $\approx 3 \mathrm{meV}$. When investigating the angular momen-

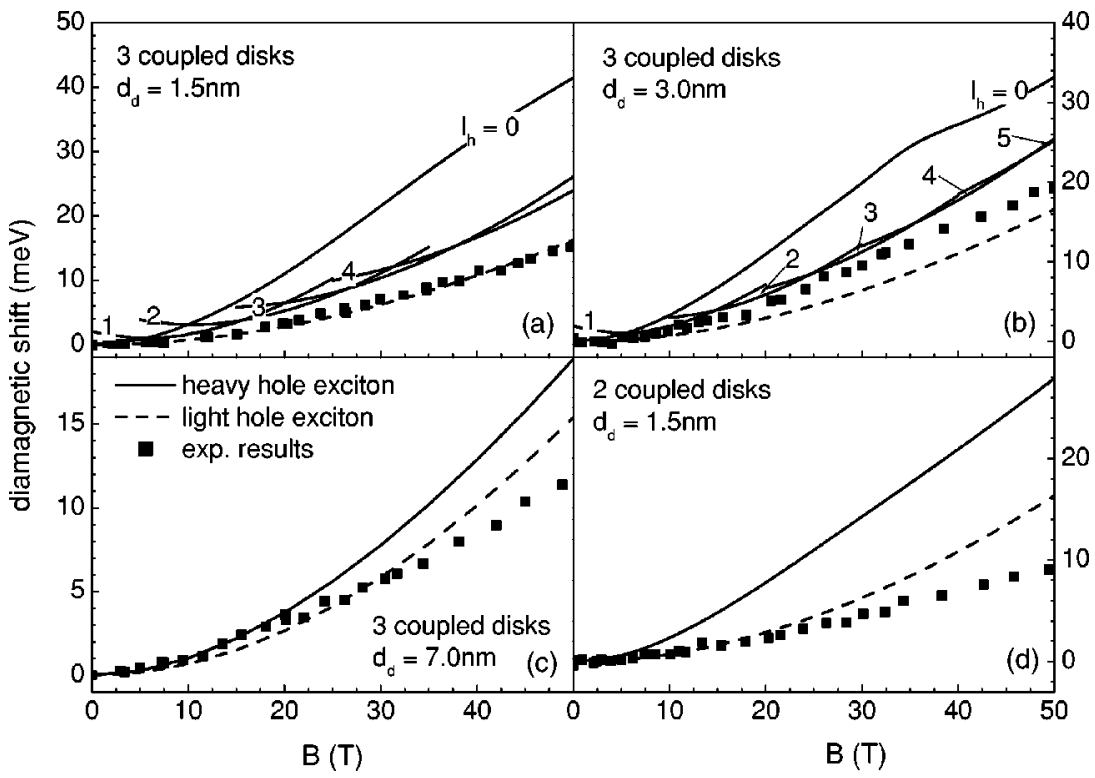

FIG. 4. Exciton diamagnetic shift as a function of the magnetic field for a system of vertically coupled identical dots with dot radius $R$ $=8 \mathrm{~nm}$ and dot thickness $d=2.5 \mathrm{~nm}$. (a) - (c) show the result for three coupled-disks with respective interdot distances $d_{d}=1.5 \mathrm{~nm}, d_{d}=3.0 \mathrm{~nm}$, and $d_{d}=7 \mathrm{~nm}$, while in (d) the diamagnetic shift is depicted for a system of two vertically coupled disks with $d_{d}=1.5 \mathrm{~nm}$. The solid and dashed curves denote, respectively, the heavy- and lighthole exciton, whereas the squares indicate the experimental result of Ref. 3. The different solid curves show the results for different heavy hole angular momentum, as indicated. 
tum transitions for the heavy hole, we find also for this system that the final heavy-hole ground state approaches the experimental result (the discrepancy between heavy hole and experiment at $B=50 \mathrm{~T}$ is $\approx 6 \mathrm{meV}$ ). However, from the transition energy, we find that the light-hole state is still the ground state. Therefore we can also conclude here that experimentally the light-hole exciton was observed, consistent with the absence of angular momentum transitions in the experimental result.

The following system under investigation consists of three vertically coupled disks with an interdot distance $d_{d}$ $=7 \mathrm{~nm}$. Comparison of the theoretical diamagnetic shift with the experimental result shows us that there is fairly good agreement for the light-hole exciton curve for magnetic fields up to $B=35 \mathrm{~T}$. For higher fields the theoretical result starts to overestimate the experimental result: at $B=50 \mathrm{~T}$ the discrepancy is $\approx 4 \mathrm{meV}$. The heavy hole in this case is confined inside the upper and lower disk, which is the reason why no angular momentum transitions occur with an increasing magnetic field. The discrepancy for the heavy hole with the experimental result $(\approx 7 \mathrm{meV})$ is larger than for the light hole. Theoretically, the light-hole exciton energy is the ground state and consequently it is this state which should be observed experimentally, in contrast to experimental results on single $\mathrm{InP} / \mathrm{GaInP}$ dots where the heavy hole was found to be the ground state. It is very likely that in the present case, there is a stronger mixing of the heavy hole into the lighthole wave function, which is probably the reason for the discrepancy with the experiment. Beyond $35 \mathrm{~T}$ the experimental diamagnetic shift has an almost linear magnetic field dependence which is clearly not observed in the theoretical curves which increase much stronger with the magnetic field. The reason for this discrepancy is yet not clear and deserves further study.

Finally, we discuss the results for a system of two vertically stacked dots. Again, we compare with the experimental result of Ref. 3. The experimentally studied system has a stack height of $7.4 \mathrm{~nm}$, which we modeled by taking two disks with $d=2.5 \mathrm{~nm}$ and interdot distance $d_{d}=1.5 \mathrm{~nm}$. The result for the diamagnetic shift is depicted in Fig. 4(d), where the solid and dashed curves denote, respectively, our results for the heavy- and the light-hole exciton and the squares denote the experimental result. The comparison between the theoretical and experimental result for the diamagnetic shift gives good agreement for the light hole up to $\approx 25 \mathrm{~T}$. For higher fields, the theoretical curve overestimates the experimental one. The discrepancy at $B$ $=50 \mathrm{~T}$ runs up to $7.5 \mathrm{meV}$. For $B>25 \mathrm{~T}$, the experimental diamagnetic shift is linear in $B$, while theoretically we still find an almost quadratic $B$-dependence indicating that the exciton size is still larger or comparable to the dot confinement potential. Also in this case we found that the light-hole exciton has a lower energy than the heavy-hole exciton and is therefore the ground state. The discrepancy between theory and experiment for $B>25 \mathrm{~T}$ is still puzzling and currently we do not have a clear explanation for this. The discrepancy may signal the breakdown of the single band approximation at high magnetic fields. This clearly deserves further investigation.

\section{CONCLUSIONS}

In this paper, we investigated the effect of strain on the band structure and exciton energy for systems of two and three vertically coupled InP/GaInP quantum disks. We considered three systems with interdot distances of, respectively, $d_{d}=1.5 \mathrm{~nm}, d_{d}=3 \mathrm{~nm}$, and $d_{d}=7 \mathrm{~nm}$.

An investigation of the influence of strain on the band structure teaches us that the light hole is always strongly confined to the barrier, i.e. outside the quantum dots. For a small interdot distance, it will be located above and below the stack of disks, whereas for large interdot distances it will be located between the disks.

In the study of the single disk, we found that the band offset for the heavy hole exhibits a potential maximum just outside the radial boundary of the disk, which increases relative to the potential maximum inside the disk for increasing disk thickness. For a stack of three vertically coupled thin disks, the potential maxima outside the radial boundary merge into one large maximum for small enough interdot distances. The strains arising from the different dots thus interact with each other. As this potential maximum is both broader and higher than the potentials inside each disk, the heavy hole prefers to be located in the barrier, at the radial boundary. For an increasing interdot distance, we find that the interaction between the strain fields decreases, and therefore also the potential maximum at the radial boundary decreases with respect to the potential inside the disks. The heavy hole will then move back into the disks. We thus find that an increase of the interdot distance can induce a type-II to type-I transition for the heavy hole, similar to the one found for a single disk but then as function of the thickness of the disk.

With regard to the exciton transition energy, we found that for all systems under consideration the light hole exciton forms the ground state. The theoretical result was found to systematically underestimate the experimental transition energies, which can be attributed mainly to the uncertainty regarding the disk and/or material parameters. Increasing the thickness of the disks will lower the theoretical exciton energies and bring it closer to the experimental results.

Results for the diamagnetic shift show for the systems of three stacked disks a fairly to very good agreement between the theoretical light-hole exciton curve and the experimental results. The agreement between theory and experiment becomes less good in the $B>20 \mathrm{~T}$ region with an increasing interdot distance. Together with the finding that the light-hole exciton forms the ground state, this allows us to conclude that experimentally the light-hole exciton was observed. No fitting of the material parameters and/or the dimensions of the dots was attempted in order to find an even better agreement between theory and experiment. For the system of two stacked dots, we find that the diamagnetic shift of the lighthole exciton agrees only well with the experimental result up to $B=25 \mathrm{~T}$. For higher fields, the theory overestimates the experimental diamagnetic shift.

In Ref. 3 it was concluded on the basis of the diamagnetic shift of the exciton energy in magnetic fields parallel and 
perpendicular to the stacks of the InP/GaInP quantum disks that the electron was located inside the dots and the hole was situated outside the dots. This conclusion agrees with our theoretical findings. But additionally we were able to refine this picture and found that (i) it is the light-hole exciton which is the exciton ground state, (ii) for the two smallest interdot spacings the hole is above and below the stack of disks. This could not be deduced from the results of Ref. 3, and (iii) only for the largest interdot spacings, the light hole is located between the dots.

\section{ACKNOWLEDGMENTS}

B.P. is a post-doctoral researcher with the Flemish Science Foundation (FWO-Vl). Part of this work was supported by the FWO-Vl, The Belgian Interuniversity Attraction Poles (IUAP), the Flemish Concerted Action (GOA) Programme, the University of Antwerp (VIS), and the European Commission GROWTH programme NANOMAT project, Contract No. G5RD-CT-2001-00545. We acknowledge interesting discussions with M. Tadić, M. Hayne, A. Zunger, A. Matulis, and J. Davies.
*Electronic address: bart.partoens@ua.ac.be

'Electronic address: francois.peeters@ua.ac.be

${ }^{1}$ For a review, see, e.g., D. Bimberg, M. Grundmann, and N. N. Ledentsov, in Quantum Dot Heterostructures (Wiley, Chichester, 1999).

${ }^{2}$ Y. Arakawa and H. Sakaki, Appl. Phys. Lett. 40, 939 (1982).

${ }^{3}$ M. Hayne, R. Provoost, M. K. Zundel, Y. M. Manz, K. Eberl, and V. V. Moshchalkov, Phys. Rev. B 62, 10324 (2000).

${ }^{4}$ M. Hayne, J. Maes, V. V. Moshchalkov, Y. M. Manz, O. G. Schmidt, and K. Eberl, Appl. Phys. Lett. 79, 45 (2001).

${ }^{5}$ L. Müller-Kirsch, R. Heitz, A. Schliwa, O. Stier, D. Bimberg, H Kirmse, and W. Neumann, Appl. Phys. Lett. 78, 1418 (2001).

${ }^{6}$ R. Heitz, N. N. Ledentsov, D. Bimberg, M. V. Maximov, A. Yu. Egorov, V. M. Ustinov, A. E. Zhukov, Zh. I. Alferov, G. E. Cirlin, I. P. Shoshnikov, N. D. Zakharov, P. Werner, and U. Gösele, Appl. Phys. Lett. 74, 1701 (1999).

${ }^{7}$ A. Polimeni, S. T. Stoddart, M. Henini, L. Eaves, P. C. Main, K. Uchida, R. K. Hayden, and N. Miura, Physica E (Amsterdam) 2, 662 (1998)

${ }^{8}$ L. R. Wilson, D. J. Mowbray, M. S. Skolnick, M. Morifuji, M. J. Steer, I. A. Larkin, and M. Hopkinson, Phys. Rev. B 57, R2073 (1998).

${ }^{9}$ O. Stier, M. Grundmann, and D. Bimberg, Phys. Rev. B 59, 5688 (1999).

${ }^{10}$ M. Brasken, M. Lindberg, D. Sundholm, and J. Olsen, Phys. Rev. B 61, 7652 (2000).

${ }^{11}$ M. Bayer, A. Schmidt, A Forchel, F. Faller, T. L. Reinecke, P. A. Knipp, A. A. Dremin, and V. D. Kulakovskii, Phys. Rev. Lett. 74, 3439 (1995).

${ }^{12}$ J. Song and S. E. Ulloa, Phys. Rev. B 52, 9015 (1995).

${ }^{13}$ C. Pryor, M.-E. Pistol, and L. Samuelson, Phys. Rev. B 56, 10404 (1997).

${ }^{14}$ M. Tadić, F. M. Peeters, and K. L. Janssens, Phys. Rev. B 65 ,
165333 (2002).

${ }^{15}$ K. L. Janssens, B. Partoens, and F. M. Peeters, Phys. Rev. B 65, 233301 (2002).

${ }^{16}$ K. L. Janssens, B. Partoens, and F. M. Peeters, Phys. Rev. B 66, 075314 (2002).

${ }^{17} \mathrm{Ph}$. Lelong, K. Suzuki, G. Bastard, H. Sakaki, and Y. Arakawa, Physica E (Amsterdam) 7, 393 (2000).

${ }^{18}$ A. B. Kalameitsev, V. M. Kovalev, and A. O. Govorov, JETP Lett. 68, 669 (1998).

${ }^{19}$ A. J. Williamson, L. W. Wang, and A. Zunger, Phys. Rev. B 62, 12963 (2000).

${ }^{20}$ M. Tadić, F. M. Peeters, K. L. Janssens, M. Korkusiński, and P. Hawrylak, J. Appl. Phys. 92, 5819 (2002).

${ }^{21}$ C. Pryor, J. Kim, L. W. Wang, A. J. Williamson, and A. Zunger, J. Appl. Phys. 83, 2548 (1998).

${ }^{22}$ J. R. Downes, D. A. Faux, and E. P. O’Reilly, J. Appl. Phys. 81, 6700 (1997).

${ }^{23}$ J. D. Eshelby, Proc. R. Soc. London, Ser. A 241, 376 (1957).

${ }^{24}$ J. H. Davies, J. Appl. Phys. 84, 1358 (1998).

${ }^{25}$ K. L. Janssens, B. Partoens, and F. M. Peeters, Phys. Rev. B 67, 235325 (2003).

${ }^{26}$ K. L. Janssens, B. Partoens, and F. M. Peeters, Phys. Rev. B 64, 155324 (2001)

${ }^{27}$ The grid consists typically of 50 points in the radial direction, of which 25 points are located in the uniform region (from 0 up to the disk radius $R$ ), and the other 25 points are located outside in the nonuniform region. In the $z$-direction, the total number of gridpoints is typically 100 , where the uniform part consists of 42 points. In the $z$-direction, the uniform region stretches over the stack of disks, i.e., the height of all disks in the stack and the interdot distances.

${ }^{28}$ S.-H. Wei and A. Zunger, Appl. Phys. Lett. 72, 2011 (1998). 